\title{
Outlier Analysis of Categorical Data using NAVF
}

\author{
D. LAKSHMI SREENIVASA REDDY ${ }^{1}$, B. RAVEENDRA BABU ${ }^{2}$, A. GOVARDHAN $^{3}$ \\ ${ }^{1}$ Rise Gandhi Group of institutions, Ongole, India \\ ${ }^{2}$ VNR VJIET, Hyderabad, India \\ ${ }^{3}$ JNTUH, Hyderabad, India \\ urdlsreddy@yahoo.com,rboghapathi@yahoo.com,govardhan_cse@yahoo.co.in
}

\begin{abstract}
Outlier mining is an important task to discover the data records which have an exceptional behavior comparing with other records in the remaining dataset. Outliers do not follow with other data objects in the dataset. There are many effective approaches to detect outliers in numerical data. But for categorical dataset there are limited approaches. We propose an algorithm NAVF (Normally distributed attribute value frequency) to detect outliers in categorical data. This algorithm utilizes the frequent pattern data mining method. It avoids problem of giving k-outliers to get optimal accuracy in any classification models in previous work like Greedy, AVF, FPOF, and FDOD while finding outliers. The algorithm is applied on UCI ML Repository datasets like Nursery, Breast cancer mushroom and bank dataset by excluding numerical attributes. The experimental results show that it is efficient for outlier detection in categorical dataset.
\end{abstract}

Keywords: Outliers, Categorical, AVF, NAVF, FPOF, FDOD

1 Introduction

Outlier analysis is an important research field in many applications like credit card fraud, intrusion detection in networks, medical field .This analysis concentrate on detecting infrequent data records in dataset.

Most of the existing systems are concentrated on numerical attributes or ordinal attributes .Sometimes categorical attribute values can be converted into numerical values. This process is not always preferable. In this paper we discuss a simple method for categorical data is presented.

AVF method is one of the efficient methods to detect outliers in categorical data. The mechanism in this method is that, it calculates frequency of each value in each data attribute and finds their probability, and then it finds the attribute value frequency for each record by averaging probabilities and selects top k- outliers based on the least AVF score. The parameter used in this method is only " $k$ ", the no. of outliers. FPOF is based on frequent patterns which are adopted from Apriority algorithm [1]. This calculates frequent patterns item sets from each object. From these frequencies it calculates FPOF score and finds the least $\mathrm{k}$ - outliers as the least FPOF scores. This method takes more time to detect outliers comparing with AVF. The parameters used in it are $\sigma$, a threshold value to decide frequent sub sets in each data object. The next method is based on Entropy score. Greedy [2] is another method to detect outliers from categorical data. The previous approaches used to detect outliers were

\section{Existing Approaches \\ Statistical based}

This method adopted a parametric model that describes the distribution of the data and the data was mostly unvaried [3, 4]. The main drawbacks of this method are difficulty of finding a correct model for different datasets and their efficiency decreases as the no. of dimensions increases [4]. To rectify this problem the Principle component method can be used. Another method to handle high dimensional datasets is to convert the data records in layers however; these ideas are not practical for more than or equal to three dimensions.

\section{Distance-Based}

Distance based methods do not make any assumptions about the distribution of the data records because they must compute the distances between records. But these make a 
high complexity. So these methods are not useful for large datasets. There are some improvements exist in the distance-based algorithms, such as Knorr's et al. [5], they have explained that apart of dataset records belong to each outlier must be less than some threshold value. Still it is an exponential on the number of nearest neighbours.

\section{Density Based}

These methods are based on finding the density of the data and identifying outliers as those lying in regions with low density. Breunig et al. have calculated a local outlier fac- tor (LOF) to identify whether an object contains sufficient neighbour around it or not[6]. They have decided a record as an outlier when the record LOF which is a user defined threshold. Papadimitriou et al. presented a similar technique called Local Correlation Integral, which deals of selecting the minimum points (min pts) in LOF through statistical methods in [7]. The density based methods have some advantages that they can detect outliers that are missed by techniques with single, global criterion methods. The terminology used in this paper is given below

Table 1. "Terminology"

\begin{tabular}{|c|c|}
\hline Term & Description \\
\hline $\mathrm{k}$ & Target number of outliers \\
\hline $\mathrm{n}$ & Number of objects in Dataset \\
\hline $\mathrm{m}$ & Number of Attributes in Dataset \\
\hline $\mathrm{d}$ & Domain of distinct values per attribute \\
\hline $\mathrm{xi}$ & i th object in Dataset ranging from 1 to $\mathrm{n}$ \\
\hline $\mathrm{Aj}$ & jth Attribute ranging from 1 to m \\
\hline $\mathrm{xij}$ & A value in xi th object which takes from domain dj of $\mathrm{j}$ th attribute $\mathrm{Aj}$ \\
\hline $\mathrm{D}$ & Item set \\
\hline $\mathrm{I}$ & Frequent Item set \\
\hline $\mathrm{F}$ & AVF score(xi) \\
\hline $\mathrm{Fi}$ & Frequency of xij value \\
\hline $\mathrm{P}(\mathrm{xij})$ & Set of frequent Item sets \\
\hline $\mathrm{FS}$ & Set of infrequent Itemsets of ithobject \\
\hline $\mathrm{IFSi}$ & Minimum support of frequent itemset \\
\hline Minsup & Support of Itemset I \\
\hline Support(I) &
\end{tabular}

\section{Algorithms}

\section{Greedy algorithm}

If any dataset consists outliers then it deviates from its original behavior and this dataset gives wrong results in any analysis. The Greedy algorithm proposed the idea of finding a small subset of the data records that contribute to eliminate the disturbance of the dataset. This disturbance is also called entropy or uncertainty. We can also define it formally as 'let us take a dataset $\mathrm{D}$ with $\mathrm{m}$ attributes A1, A2--- Am and $\mathrm{d}(\mathrm{Ai})$ is the domain of distinct values in the variable $\mathrm{Ai}$, then the entropy of single attribute $\mathrm{Aj}$ is

$$
\begin{array}{r}
E(A j)=-\sum p(x) \log 2(p(x)) \\
x \varepsilon d(A j)
\end{array}
$$

Because of all attributes are independent to each other, Entropy of the entire dataset

$\mathrm{D}=\{\mathrm{A} 1, \mathrm{~A} 2$------- $\mathrm{Am}\}$ is equal to the sum of the entropies of each one of the $m$ attributes, and is defined as follows

$\mathrm{E}(\mathrm{A} 1, \mathrm{~A} 2---\mathrm{Am})=\mathrm{E}(\mathrm{A} 1)+\mathrm{E}(\mathrm{A} 2)+---\mathrm{E}(\mathrm{Am})(2)$

When we want to find entropy the Greedy algorithm takes k outliers as input [2]. All records in the set are initially designated as non-outliers. Initially all attribute value's frequencies are computed and using these frequencies the initial entropy of the dataset is calculated. Then, Greedy algorithm scans $\mathrm{k}$ times over the data to determine the top $\mathrm{k}$ 
outliers keeping aside one non-outlier each time. While scanning each time every single non-outlier is temporarily removed from the dataset once and the total entropy is recalculated for the remaining dataset. For any nonoutlier point that results in the maximum decrease for the entropy of the remaining dataset is the outlier data-point removed by the algorithm. The Greedy algorithm complexity is $\mathrm{O}(\mathrm{k} * \mathrm{n} * \mathrm{~m} * \mathrm{~d})$, where $\mathrm{k}$ is the required number of outliers, $\mathrm{n}$ is the number of objects in the dataset $\mathrm{D}, \mathrm{m}$ is the number of attributes in $\mathrm{D}$, and $\mathrm{d}$ is the number of distinct attribute values, per attribute. Pseudo code for the Greedy Algorithm is as follows

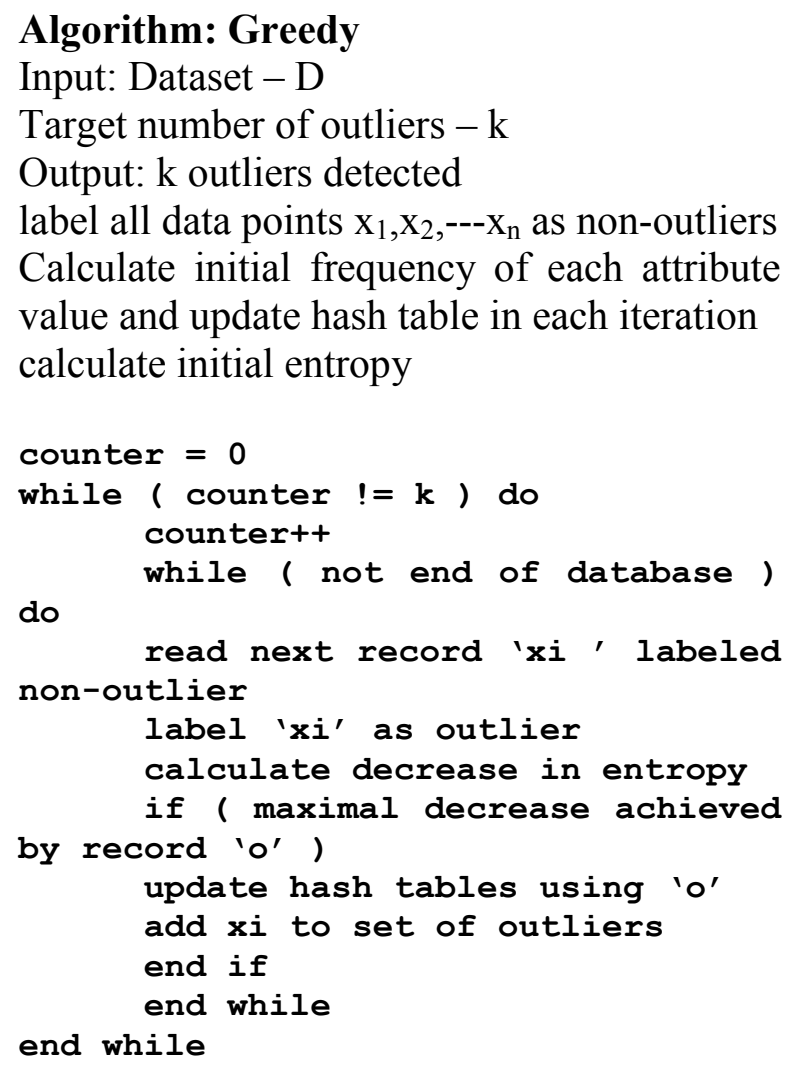

However entropy needs $\mathrm{k}$ as input and need to find number of outliers more times to get optimal accuracy of any classification model.

\section{AVF algorithm}

The algorithm discussed above is linear with respect to data size and it needs k-scans each time. The other models also exist which are based on frequent item set mining (FIM) need to create a large space to store item sets, and then search for these sets in each and every data point . These techniques can become very slow when we select low threshold value to find frequent item sets from dataset

Another simpler and faster approach to detect outliers that minimizes the scans over the data and does not need to create more space and more

Search for combinations of attribute values or item sets is Attribute Value Frequency (AVF) algorithm. An outlier point xi is defined based on the AVF Score below:

$\operatorname{AVF}$ Score $(x i)=F_{i}=\frac{1}{m} \sum_{j=1}^{m} f(x i j)$

In this approach [1] again we need to find koutliers many times to get optimal accuracy of any classification model. Pseudo code for the AVF Algorithm is as follows

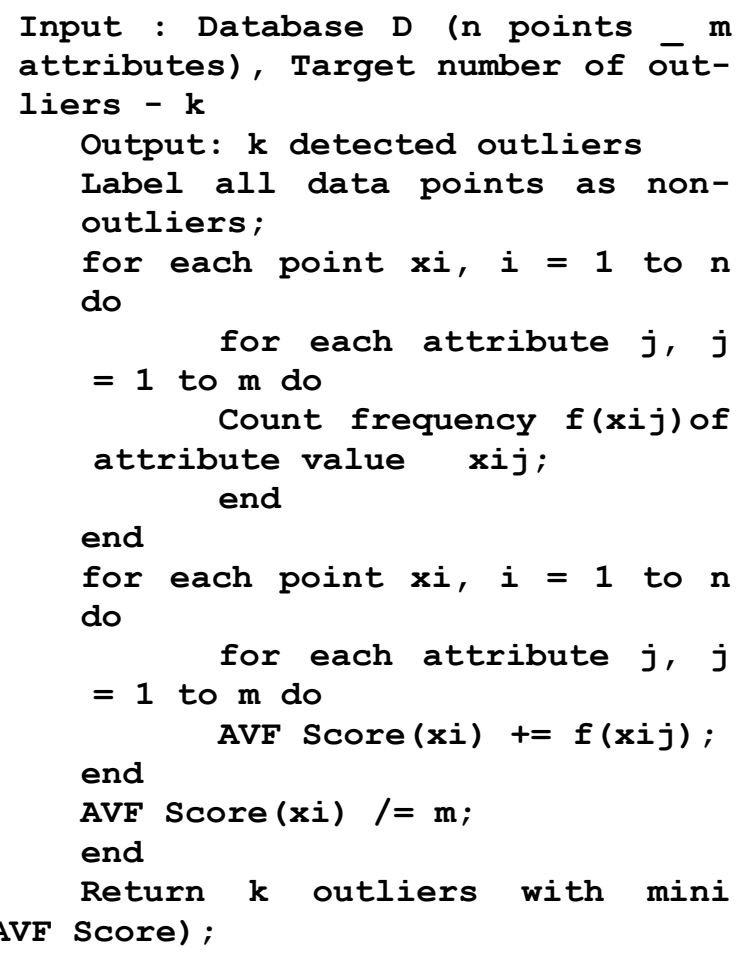

The AVF algorithm complexity is lesser than Greedy algorithm since AVF needs only one scan to detect outliers. The complexity is $\mathrm{O}$ $(\mathrm{n} * \mathrm{~m})$. It needs ' $\mathrm{k}$ ' value as input. In FPOF [8] this has discussed frequent pattern based outlier detection, in this too k-value and another parameter ' $\sigma$ ' are required as threshold. This also discussed about frequent pattern based method to find infrequent object, in 
this too it requires k-value, and another parameter ' $\sigma$ ' as input.

\section{N AVF algorithm}

This proposed model (NAVF) has been defined as an optimal number of outliers in a single instance to get optimal precision in any classification model with good precision and low recall value. This method calculates ' $\mathrm{k}$ ' value itself based on the frequency. Let us take the data set ' $D$ ' with ' $m$ ' attributes $A 1$, A2----- Am and d (Ai) is the domain of distinct values in the variable $\mathrm{Ai} . \mathrm{k}_{\mathrm{N}}$ is the number of outliers which are normally distributed. To get ' $\mathrm{k}_{\mathrm{N}}$ ' this model used Gaussian theory. If any object frequency is less than "mean-3 S.D" then this model treats those objects as outliers. This method uses AVF score formula to find AVF score but no kvalue is required. Let $\mathrm{D}$ be the Categorical dataset, contains ' $n$ ' data points, $x_{i}$, where $i=$ 1 ...n. If each datapoint has ' $m$ ' attributes, we can write $\mathrm{x}_{\mathrm{i}}=\left[\mathrm{x}_{\mathrm{i} 1}, \ldots . . \mathrm{x}_{\mathrm{il}}, \ldots . . \mathrm{x}_{\mathrm{im}}\right]$, where $\mathrm{x}_{\mathrm{il}}$ is the value of the $1_{\text {th }}$ attribute of $x_{i}$.

\section{Algorithm}

Input: Dataset - D,

Output: K detected outliers.

\section{Step 1: Read data set D}

Step 2: Label all the Data points as nonoutliers

Step 3: calculate normalized frequency of each attribute value for each point $\mathrm{x}_{\mathrm{i}}$

Step 4: calculate the frequency score of each record $\mathrm{x}_{\mathrm{i}}$ as, Attribute Value Frequency of $\mathrm{x}_{\mathrm{i}}$ is:

$$
\operatorname{AVF} \text { Score }\left(x_{i}\right)=F_{i}=\frac{1}{m} \sum_{j=1}^{m} f(x i j)
$$

Step 5: compute $\mathrm{N}$-seed values $\mathrm{a}$ and $\mathrm{b}$ as $\mathrm{b}=$ mean $\left(\mathrm{x}_{\mathrm{i}}\right), \mathrm{a}=\mathrm{b}-3^{*}$ std $\left(\mathrm{x}_{\mathrm{i}}\right)$, if $\max \left(\mathrm{F}_{\mathrm{i}}\right)>$ $3 *$ std $\left(\mathrm{F}_{\mathrm{i}}\right)$

Step 6: If $\mathrm{Fi}<\mathrm{a}$, then declare $\mathrm{x}_{\mathrm{i}}$ as outlier Step 7: return $K_{N}$ detected outliers.

Table 2. "Outliers of Nursery Data"

\begin{tabular}{|c|c|c|c|c|}
\hline & \multirow{2}{*}{ Actual outliers } & \multirow{2}{*}{ Total records } & \multicolumn{2}{|c|}{ NAVF } \\
\cline { 3 - 5 } & & & True positives & False positives \\
\hline 1-in-2 & 956 & 5276 & 44 & 1 \\
\hline 1 -in-5 & 382 & 4702 & 132 & 1 \\
\hline 1 -in-8 & 238 & 4558 & 238 & 0 \\
\hline 1-in-10 & 190 & 4570 & 190 & 0 \\
\hline
\end{tabular}

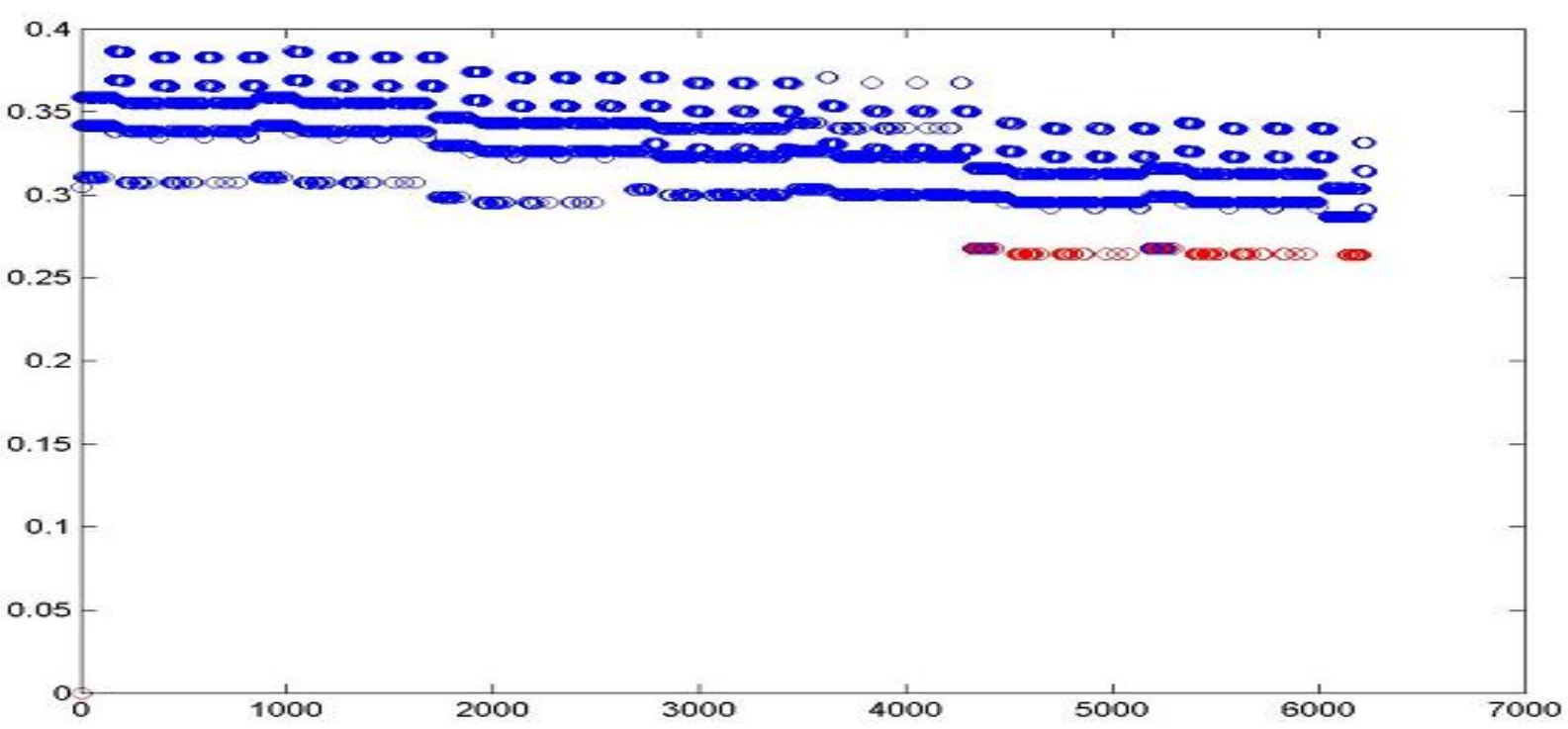

Fig. 1. "Outliers in Nursery Data" 

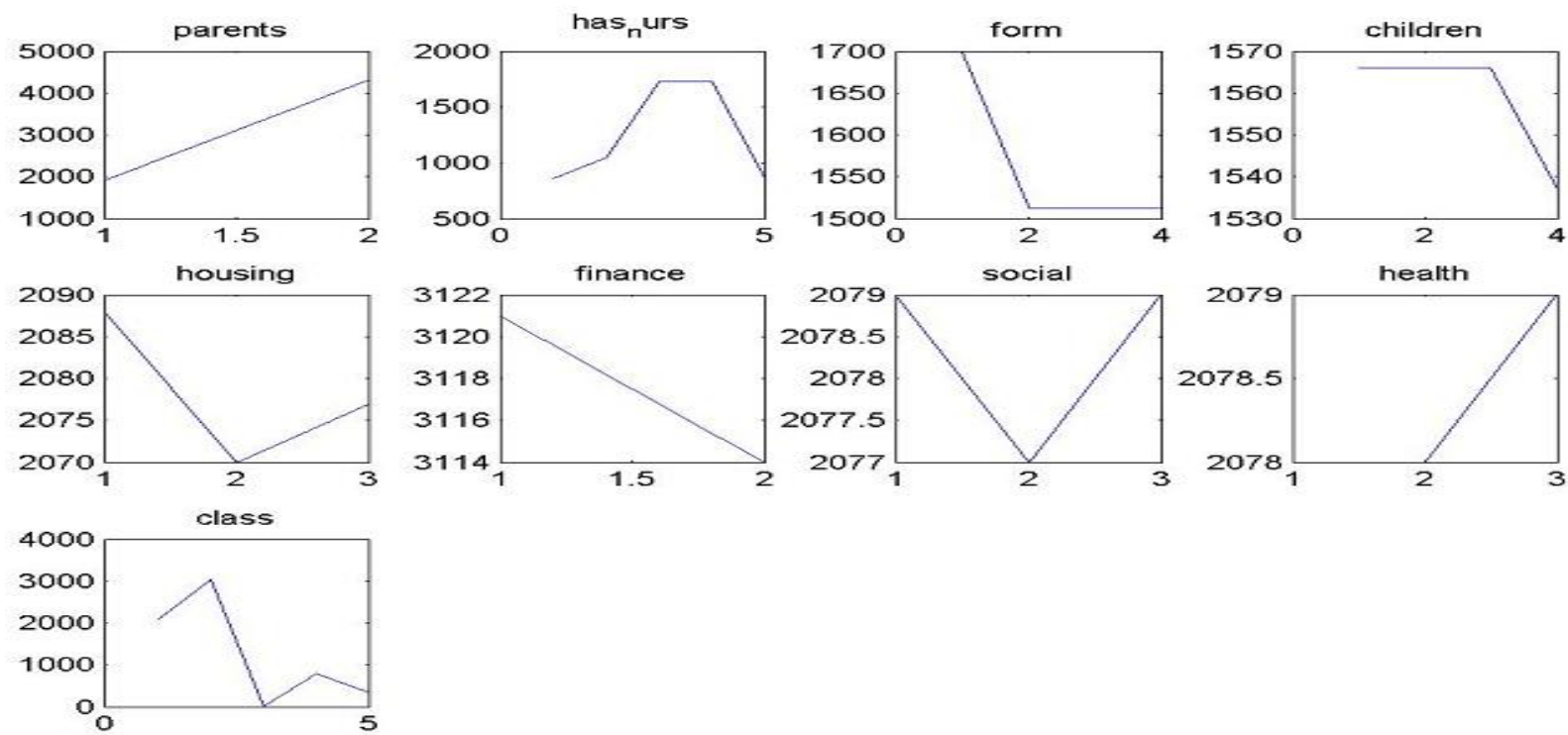

Fig. 2. "Nursery Data Frequency for each Attribute values"

\section{Experimental Results}

In this paper this model has been applied on Breast Cancer, Nursery data and Bank marketing data from UCI Machine repository [9]. This method has implemented the approach of using MATLAB tool. We ran our experiments on a workstation with a Pentium( $\mathrm{R}$ ) $\mathrm{D}, 2.80 \mathrm{GHz}$ Processor and $1.24 \mathrm{~GB}$ of RAM.

Nursery data consists of nine attributes and 6236 records. This data divided into two parts based on parent attribute, first part contains 4320 records with usual parent type, and second part contain 1916 records with pretentious parent type which is used as outliers in our experiment. In first iteration 956 sample records are selected randomly using Clementine tool; from each two records one is selected. These 956 records are mixed up with part one and applied normally distributed AVF to get outliers. The found outliers are given in Table 2. Similarly in the next iteration 382 records are selected randomly as one record from each five records and mixed up with first part and applied the same process. The results are given in the Table 2. Similarly one record is selected from each eight records and ten records and repeated the same process. This method has been implemented on Nursery dataset, Breast cancer and Bank dataset which are taken from UCI Machine learning repository [9]. This method compared with different number of outliers from each sample. Comparison graph is given in Figure 3. For Nursery Data Figure 1 shows the frequency of different attribute values and their structure.

Figure 2 shows the outliers which are appeared in red colour for the Nursery Data

These red collared points are under "Mean3SD" line which we can observe in the Figure 2. This Figure 2 is drawn by MATLAB tool for the data taken by the 1-in-2 sample method.

In the first sample from nursery the NAVF model found out only $4.60 \%$ of outliers from 956 outliers which are mixed up with 4320 records which totals to 5276 records.. In the next sample of 382 records, $34.8 \%$ of correct outliers are found by NAVF. For the sample of 238 records NAVF found 239 outliers in which 238 are correct, which means that NAVF model found $100 \%$ outliers correctly. Similarly NAVF model found $100 \%$ outliers in the sample of 190 records (as outliers) mixed up with 4320 records in part one. 
Table 3. "Breast Cancer Results"

\begin{tabular}{|c|c|c|c|c|}
\hline \multirow{2}{*}{$\begin{array}{c}\text { Sample } \\
\text { method }\end{array}$} & \multirow{2}{*}{ Actual outliers } & \multirow{2}{*}{ Total records } & \multicolumn{2}{|c|}{ NAVF } \\
\cline { 3 - 5 } & & & True positives & False positives \\
\hline 1 -in-2 & 119 & 577 & 35 & 0 \\
\hline $1-$ in-5 & 48 & 506 & 9 & 0 \\
\hline 1 -in-8 & 29 & 487 & 9 & 0 \\
\hline 1 -in-10 & 23 & 481 & 14 & 1 \\
\hline
\end{tabular}

In case of breast cancer dataset, correct outliers found by NAVF model did not touch $100 \%$. In breast cancer data 119, 48, 29, 23 outliers are selected respectively using 1 -in2, 1 -in-5, 1-in-8, 1-in-10 sampling from benign breast cancer. NAVF found $35,9,9$, and 14 correct and 0, 0, 0, 1 wrong from 119, 48,
29, 23 outliers. The results are given in Table 3. The comparison of outlier detection is shown in Figure 4.

In Bank marketing data, only categorical attributes are selected and 2644, 1027, 661, 528 outliers are selected respectively using 1in-2, 1 -in-5, 1-in-8, 1-in-10 sampling

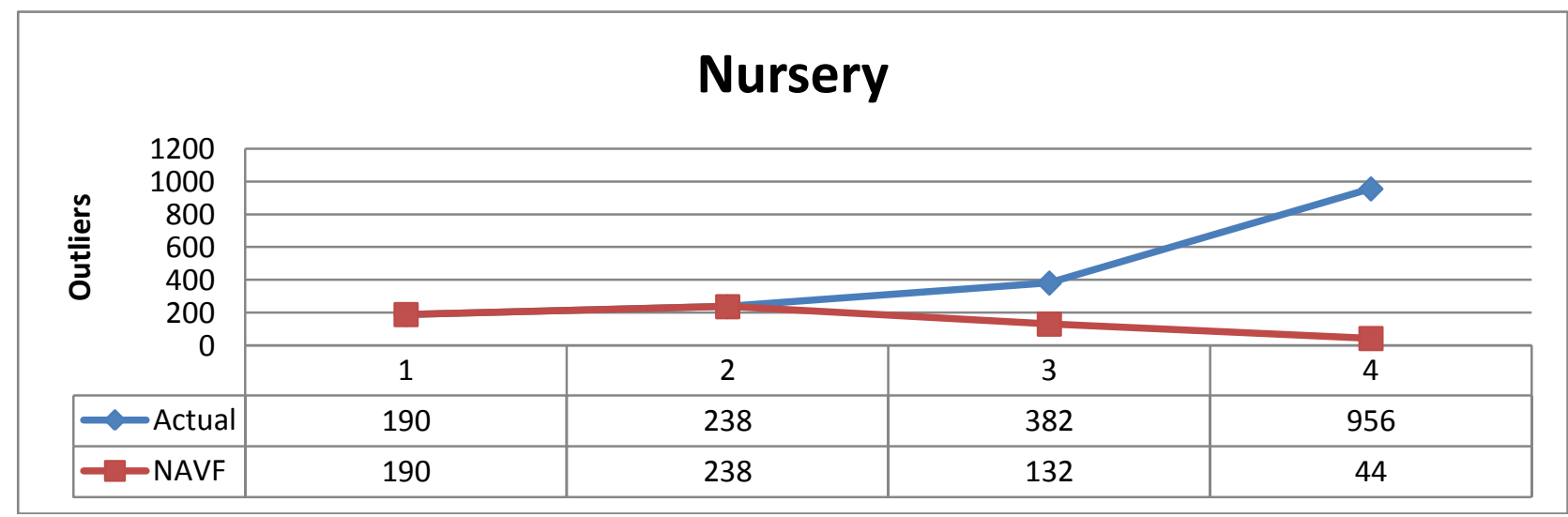

Fig. 3. "Outliers in Nursery Data"

Table 4. "Bank Data Results"

\begin{tabular}{|c|c|c|c|c|}
\hline \multirow{2}{*}{ Sample method } & \multirow{2}{*}{ Actual outliers } & \multirow{2}{*}{ Total records } & \multicolumn{2}{|c|}{ NAVF } \\
\cline { 3 - 5 } & & 39922 & True positives & False positives \\
\hline 1 -in-2 & 2644 & 39922 & 274 & 100 \\
\hline 1 -in-5 & 1027 & 39922 & 198 & 168 \\
\hline 1 -in-8 & 661 & 39922 & 126 & 202 \\
\hline 1 -in-10 & 528 & & 213 \\
\hline
\end{tabular}

method from the attribute Y="yes" and applied the same process as above. In this data NAVF found 274, 198, 152, 126 correct outliers and 100, 168, 202, 213 wrong outliers from the random sample of $2644,1027,661$, 528 outliers taken by 1 -in-2, 1 -in-5, 1-in-8, 1-in-10 sampling method. The found outliers are arranged in Table 4 . In this table the true positives and false positives found by our model are arranged.

Similarly we applied the same process for all samples in banking data. The results are summarized in the Table 4 and its graph is given in Figure 5. Different classification models are tested for accuracy of the bank dataset after deleting the outliers. Different classifiers are tested on 1-in-5 sample data which contain 39922 original records mixed up with 1027 outliers. The NAVF model has found 366 records as outliers in which 198 are correct outliers and 168 are wrong outlier (original records). When Neural network, C5, CRT, QUEST, CHAID Linear Regression, Decision Logic Classifiers are applied on the 
above sample data, the classifiers have given the accuracies as given in the Table 5 .

Different classifiers are applied on the remaining dataset after deleted the outliers by
NAVF model. All classifiers have given good results for NAVF. Only the decision logic classifier gave very less accuracy (37.058) by NAVF.

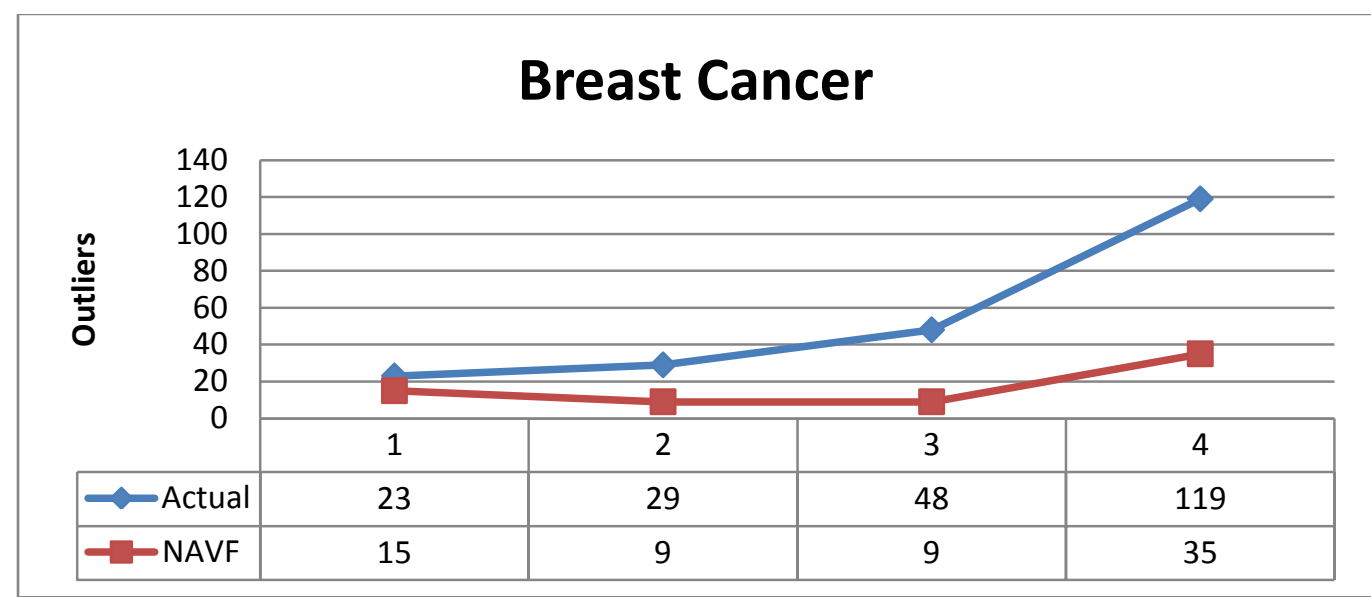

Fig. 4. "Outliers in Breast Cancer Data"

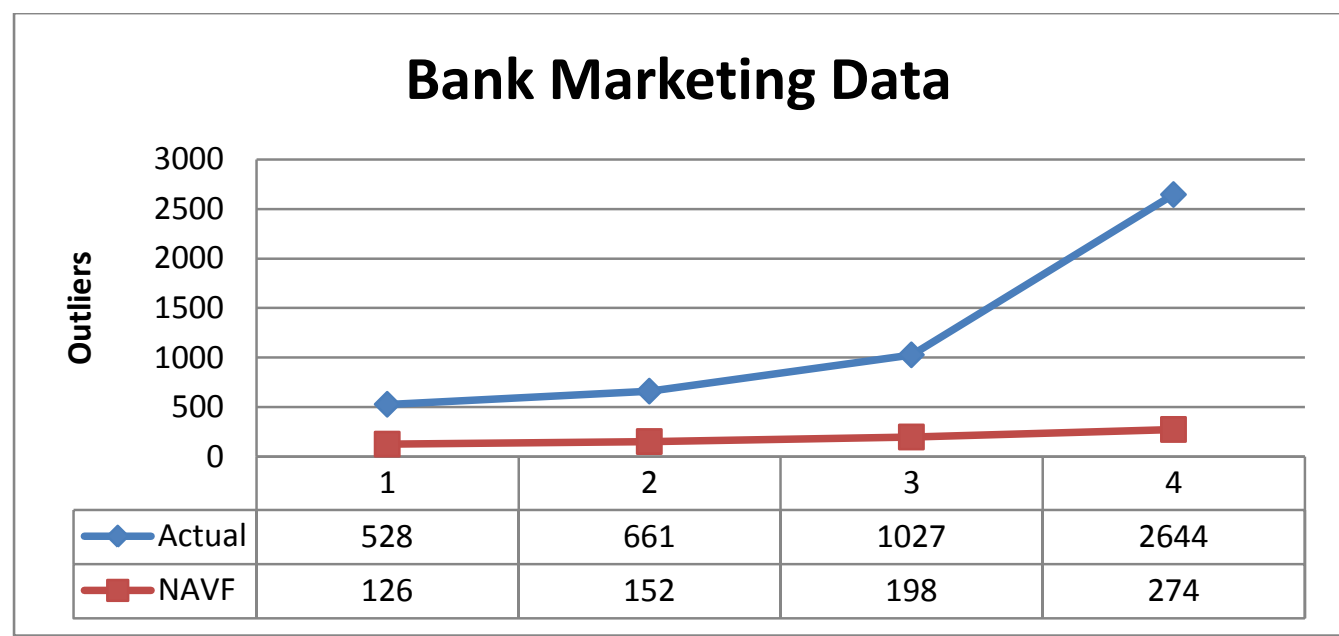

Fig. 5. "Outliers in Bank Marketing Data"

Table 5. "Classifiers Results on Bank Data"

\begin{tabular}{|c|c|c|c|c|c|}
\hline Classifier & NAVF & $\mathbf{K}=\mathbf{1 0 0}$ & $\mathbf{K}=\mathbf{3 5 4}$ & $\mathbf{K = 3 7 0}$ & $\mathbf{K = 5 0 0}$ \\
\hline NN & 98.735 & 97.581 & 97.878 & 97.885 & 97.994 \\
\hline C5 & 98.735 & 97.581 & 97.881 & 97.885 & 97.994 \\
\hline CRT & 98.735 & 97.581 & 97.881 & 97.885 & 97.994 \\
\hline QUEST & 98.735 & 97.581 & 97.881 & 97.885 & 97.994 \\
\hline DL & 37.058 & 95.445 & 92.933 & 92.94 & 92.339 \\
\hline CHAID & 98.735 & 97.581 & 97.881 & 97.885 & 97.994 \\
\hline LR & 98.735 & 97.581 & 97.881 & 97.882 & 97.994 \\
\hline
\end{tabular}

\section{Conclusion and Future Work}

To sum up, this proposed method gives the optimal number of outliers ' $\mathrm{K}_{\mathrm{N}}$ '. In existing models it is mandatory to give the number of outliers to find them .While taking the num- ber of outliers sometimes the original data may be missed. If any classifier modelled using this data, wrong classifiers may be modelled. In future there is a possibility of checking the precision and recall values of each 
model with the existing models. The same method can also be applied on mixed type of dataset.

\section{Reference}

[1] M. E. Otey, A. Ghoting, and and A. Parthasarathy, "Fast Distributed Outlier Detection in Mixed-Attribute Data Sets," Data Mining and Knowledge Discovery

He, Z., Deng, S., Xu, X., "A Fast Greedy algorithm for outlier mining", Proc. of PAKDD, 2006.

[2] I. S. Jacobs and C. P. Bean, "Fine particles, thin films and exchange anisotropy," in Magnetism, vol. III, G. T. Rado and H. Suhl, Eds. New York: Academic, 1963, pp. 271-350.

[3] P. Tan, M. Steinbach, and V. Kumar, Introduction to Data Mining: Pearson Addison-Wesley, 2005

[4] E. Knorr, R. Ng, and V. Tucakov, "Distance-based outliers: Algorithms and applications," VLDB Journal, 2000.

M. M. Breunig, H.-P. Kriegel, R. T. Ng, and J. Sander, "LOF: Identifying density based local outliers," presented at ACM SIGMOD International Conference on Management of Data, 2000

[5] S. Papadimitriou, H. Kitawaga, P. Gibbons, and C. Faloutsos, "LOCI: Fast outlier detection using the local correlation integral," presented at International Conference on Data Engineering, 2003

[6] Z. He, X. Xu, J. Huang, and S. Deng, "FP-Outlier: Frequent Pattern Based Outlier Detection", Computer Science and Information System (ComSIS'05)," 2005

[7] S. Wu and S. Wang, "InformationTheoretic Outlier Detection for LargeScale Categorical Data, IEEE Transactions on Knowledge Engineering and Data Engineering,2011

[8] A. Frank, \& A. Asuncion, (2010). UCI Machine Learning Repository [http://archive.ics.uci.edu/ml]. Irvine, CA: University of California, School of Information and Computer Science.
[9] B. Yu, M. Song, L. Wang "Local Isolation Coefficient-Based Outlier Mining Algorithm", International Conference on Information Technology and Computer Science 2009

[10] M. Gebski, A. Penev Raymond, K. Wong "Grouping Categorical Anomalies" IEEE/WIC/ACM International Conference on Web Intelligence and Intelligent Agent Technology2008

[11] E. M"uller, I. Assent, U Steinhausen , Thomas Seidl "Out Rank ranking outliers in high dimensional data" ICDE Workshop 2008

[12] Z. Huang. Extensions to the k-Means Algorithm for Clustering Large Data Sets with Categorical Values. Data Mining and Knowledge Discovery, 1998

[13] C. R. Palmer, C. Faloutsos." Electricity Based External Similarity of Categorical Attributes." In Proc. of the 7th Pacific-Asia Conference on Advances in Knowledge Discovery and Data Mining (PAKDD'03), 2003

[14] S. Q. Le, T. B. Ho.” A Conditional Probability Distribution-Based Dissimilarity Measure for Categorical Data". In: Proc. of the 8th Pacific-Asia Conference on Advances in Knowledge

[15] Discovery and Data Mining (PAKDD'04), 2004

[16] V. Cheng, C. H. Li, J. T. Kwok, C-K. Li. "Dissimilarity learning for nominal data. Pattern Recognition", 2004

[17] S-G. Lee, D-K. Yun. "Clustering Categorical and Numerical Data : A New Procedure Using Multi-dimensional Scaling". International Journal of Information Technology and Decision Making",2003

[18] C. Li, G. Biswas. "Un supervised learning with mixed numeric and nominal data”. IEEE Transactions on Knowledge and Data Engineering, 2002

[19] Z. Huang, M. K. Ng. "A fuzzy kmodes algorithm for clustering categorical data. IEEE Transaction on Fuzzy Systems", 1999

[20] Mahoney, M.V.and Chan, P.K.. "Learning non stationary models of normal network traffic for detecting novel 
attacks". Proc. of the ACM SIGKDD International Conference on Knowledge Discovery and Data Mining. 2002.

[21] Palpanas, T., Papadopoulos, D., Kalogeraki, V., and Gunopulos, D. "Distributed deviation detection in sensor networks". SIGMOD Record, 2003.

[22] Knorr, E. and Ng, R.T. "Algorithms for mining distance-based outliers in large datasets", Proc. of the International Conference on Very Large Databases. 1998.

[23] Lazarevic, A., Ertoz, L.,Ozgur, A.,Kumar,V., and Srivastava, J.."Acomparative study of outlier detection schemes for network intrusion detection". Proc. of the SIAM International Conference on Data Mining 2003.

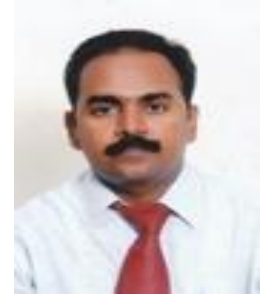

Mr. D. LAKSHMI SREENIVASA REDDY obtained his Master Degree from JNT university, Hyderabad, and doing PhD in the same University in Computer Science \& Engineering. He is currently heading the Department of Computer Science \& Engineering, Rise Gandhi Groups of Institutions, Ongole. He has 11 years of teaching experience. His Research interest is in Data mining.

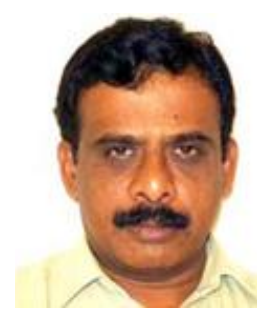

Dr. B. RAVEENDRA BABU obtained his Masters in Computer Science and Engineering from Anna University, Chennai. He received his Ph.D. in Applied Mathematics at S.V University, Tirupati. He is currently heading the Department of Computer Science \& Engineering, RVR \& JC College of Engineering, Guntur. He has 27 years of teaching experience. He has several publications to his credit. His research areas of interest include VLDB, Image Processing, Pattern analysis and Wavelets.

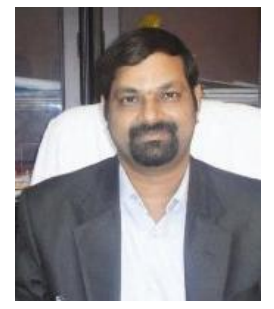

Dr. A. GOVARDHAN did his BE in Computer Science and Engineering from Osmania University College of Engineering, Hyderabad in 1992, M. Tech from Jawaharlal Nehru University(JNU), Delhi in 1994 and he earned his $\mathrm{PhD}$ from Jawaharlal Nehru Technological University, Hyderabad (JNTUH) in 2003.He joined Jawaharlal Nehru Technological University in the year 1994 as an Assist. Prof., became Associate Professor in 1999 and Professor in 2006. 